\title{
Explanatory Model Interview Catalogue (EMIC): Framework for Comparative Study of Illness
}

\section{Citation}

Weiss, M. 1997. “Explanatory Model Interview Catalogue (EMIC): Framework for Comparative Study of Illness." In Transcultural Psychiatry 34, no. 2: 235-263. doi:10.1177/136346159703400204.

\section{Published Version}

doi:10.1177/136346159703400204

\section{Permanent link}

http://nrs.harvard.edu/urn-3:HUL.InstRepos:35642370

\section{Terms of Use}

This article was downloaded from Harvard University's DASH repository, and is made available under the terms and conditions applicable to Other Posted Material, as set forth at http:// nrs.harvard.edu/urn-3:HUL.InstRepos:dash.current.terms-of-use\#LAA

\section{Share Your Story}

The Harvard community has made this article openly available.

Please share how this access benefits you. Submit a story.

\section{Accessibility}


Explanatory Model Interview Catalogue (EMIC):

Framework for Comparative Study of Illness Experience

Mitchell Weiss, M.D., Ph.D.

Professor and Head

Department of Public Health and Epidemiology

Swiss Tropical Institute, University of Basel

Address for Correspondence:

Department of Public Health and Epidemiology Swiss Tropical Institute

Socinstrasse 57 ,

$\mathrm{CH}-4002$ Basel

SWITZERLAND

Tel: +41 $61 \quad 284 \quad 8284$

Fax: +4161 2717951

E-Mail: weissm@ubaclu.unibas.ch

April 8, 1997 


\section{Explanatory Model Interview Catalogue (EMIC): Framework for Comparative study of Illness Experience}

\section{Abstract}

The Explanatory Model Interview Catalogue (EMIC) refers to a collection of locally adapted explanatory model interviews rooted in a common framework. Efforts to develop the EMIC were motivated by research experience in cultural psychiatry and tropical medicine that demonstrated a need to integrate epidemiological and anthropological research methods more effectively. Various adaptations of the EMIC framework have produced semistructured interviews based on an operational formulation of an illness explanatory model that systematically clarifies the experience of illness from the point of view of the people who are directly affected. Patterns of distress, perceived causes, preferences for help seeking and treatment, and general illness beliefs constitute a framework for the operational formulation of the illness explanatory model. Data sets generated from these EMIC interviews typically include quantitative variables and qualitative prose, which are cross-referenced for analysis to clarify key features and answer important questions about illness experience and its practical implications. This review discusses the development and structure of the EMIC, the adaptation of particular explanatory model interviews, the analysis of data obtained from these interviews, the scope of research they have addressed, and next steps in the development of the EMIC. 


\section{Explanatory Model Interview Catalogue (EMIC): Framework for Comparative Study of Illness Experience}

Cultural beliefs and practices affect nearly all aspects of psychiatry, especially assessment and diagnosis, illness behavior and help seeking, mutual expectations of interactions between patients and practitioners, perceived quality of care, and the design of culturally appropriate psychotherapies. Fundamental questions about the cultural underpinnings of psychopathology, as well as practical concerns about the underutilization and ineffectiveness of mental health services for cultural minorities and for patients in non-Western countries affect health planning and challenge the field of cross-cultural psychiatry in international and increasingly multicultural local settings (Desjarlais, Eisenberg, Good \& Kleinman, 1995; Kareem \& Littlewood, 1992; Littlewood \& Lipsedge, 1989). Even when the sociocultural backgrounds of patients and health professionals are similar, without a framework to guide them clinicians may fail to appreciate or even to inquire about the significance of their patients' distinctive ideas and illness experience.

Although other areas of psychiatry have been invigorated by research agendas rooted in theoretical frameworks with a clear relationship between significant questions and the methods needed to answer them, a coherent methodology for cross-cultural inquiry focusing on study of patients' illness experiences and their implications has remained elusive (Rogler, 1992; Rogler, 1989; Weiss \& Kleinman, 1988; Dohrenwend \& Dohrenwend, 1974; Sartorius, 1973). Epidemiological comparisons, which may neglect the complexity of illness experience, and anthropological study, which may lack the analytic power to test hypotheses concerning relationships between risk factors and disease outcomes, each have limitations, indicating a need for research methods that benefit from complementary aspects of the two disciplines.

Efforts to develop the Explanatory Model Interview Catalogue (EMIC) aimed to provide a method for cross-cultural research that incorporates advances in psychiatric epidemiology associated with semi-structured interviews and operational guidelines for recording and analyzing data (Robins, Wing, Wittchen, Helzer, Babor, Burke, Farmer, Jablenski, Pickens, Regier, Sartorius \& Towle, 1988; Riskind, Beck, Berchick, Brown \& Steer, 
1987; Kraemer, Pruyn, Gibbons, Greenhouse, Grochocinski, Waternaux \& Kupfer, 1987), as well as advances in medical anthropology and the socalled new cross-cultural psychiatry (Littlewood, 1991; Kleinman, 1988a; Kleinman, 1977). Development of the EMIC was also motivated by recognition of the inadequacy of assumptions based on experience with patients from the majority cultures in North America and Western Europe as guidelines for clinical practice and mental health planning in developing countries and multicultural societies (Jadhav, 1995).

The EMIC refers to a framework for semi-structured interviews based on a particular operational formulation of the concept of illness explanatory models and a set of explanatory model interviews based on that framework. It differs from standard instruments of psychiatric epidemiology in two respects: the EMIC is a catalogue of explanatory model interviews based on an adaptable, operational framework and the experience acquired from their use; it is not a single instrument for generic use irrespective of particular clinical and cultural contexts. Also, unlike most psychiatric research instruments, which were developed in North America and Western Europe, the initial EMIC interviews were developed in India and subsequently adapted for use in North America, Western Europe, Africa, and East Asia.

\section{Conceptual Basis of the EMIC}

The terms emic and etic distinguish frameworks for social analysis, the first rooted in ideologies of local communities (emic) and the second based on the ideology of professionals outside these local communities (etic) (Headland, Pike and Harris, 1990). An epidemiological study of professionally defined categories, for example, schizophrenia, depression, or social class, is etic. An ethnographic study that focuses on local categories of meaning, on the other hand, for example, the particulars of humoral balance, magico-religious forces, or caste structure (in India), may be considered emic. This distinction of emic and etic perspectives was formulated by Kenneth Pike in 1954 based on principles derived from comparative linguistics (Pike, 1967). Subsequent comparable formulations, applied to medical anthropology and social medicine, of the underlying dichotomy distinguishing local insider and professional outsider perspectives include the relationship between explanatory models and 
clinical diagnoses and the relationship between illness and disease (Hahn, 1984; Kleinman, 1980; Eisenberg, 1977).

These concepts and the opposition represented by emic and etic perspectives provide a framework for understanding the relationship between biomedical models and patients' experiences. Although much work in the field of medical anthropology refers implicitly or explicitly to this framework, the relationship between local and professional ideas is not necessarily dichotomous or even clear. Professional medical ideologies influence popular ideas about illness, either subtly from patients' interactions with medical professionals or overtly as a result of health education, medical writing, and other communications that target lay audiences. Explanatory models refer therefore both to independent, locally rooted concepts as well as to local interpretations of professional ideas; prevailing ideas about illness are not necessarily easy to classify as emic or etic.

This ambiguity reflects not only the influence of professional ideas on popular experience but also professional versions of popular ideas about illness, such as the adverse impact of stress. In non-Western cultures, such as India, ideas of humoral balance--e.g., heat and cold, or wind (vāta), bile (pitta), and phlegm ( popular cultures and the formal medical traditions that it is impossible to associate them with one or the other exclusively. Although explanatory model interviews based on the EMIC framework aim to elicit explanatory models of subjects in their own terms, which emphasizes the emic component, this emphasis can only be relative, not absolute.

Kleinman's concept of explanatory model, initially developed in clinic settings, focused on the meaning of illness and the sense people make of it. Although the explanatory model framework has motivated considerable research in psychiatry, medicine, and medical anthropology, and it defined an approach for cultural study of illness that motivated development of the EMIC, it is not the only conceptual formulation of illness meanings for medically applied social sciences or medical anthropology. Other frameworks for studying health, illness, and their sociocultural contexts suggest alternative research paradigms to the cognitive orientation of patients' illness explanatory models. Critical medical anthropology, concerned mainly with the impact on health and health 
care of differences in class, power, and status, provides such an alternative framework for activities in the field. Though initially considered more academic, this research may also address practical matters of policy (Singer, 1995). Ethnographic, life history, and observational methods in local communities as well as analysis of public policy also provide valuable alternatives to semi-structured interviews like the EMIC or field-based epidemiological studies as an approach to policy-relevant health research. These methods have been especially well suited for addressing the problems of poor and disadvantaged populations, and several studies of homelessness make this point very well (Williams, 1995; Pinder, 1994; Mathieu, 1993; Koegel, 1992; Rosenthal, 1991). Although the EMIC is rooted in the explanatory model framework with all of its advantages and limitations, different methods are clearly better suited to different tasks and in any event need to be used thoughtfully.

Various researchers have elaborated other approaches to the study of illness meanings. In contrast to Kleinman's formulation of illness explanatory models, rooted in clinical experience with patients, Eisenbruch (1990) consulted George Murdock's (1980) worldwide survey of illness models as a source of cultural illness categories. Eisenbruch's cross-cultural research analyzed psychiatric illness beliefs of non-patient subjects, rather than studying clinical samples with reference to course and outcome. Studies of illness behavior, introduced by David Mechanic in the 1950s, recognized individual variation in sociocultural meanings of illness as important determinants of help-seeking choices, coping, mobilization and use of social supports, and quality of life (Mechanic, 1995). Findings from this research highlighted the distinctive emphasis among patients on pain, discomfort, and well-being, in contrast to the emphasis of clinicians on differential diagnosis and the features of recognized clinical disorders. Mechanic's work also considered the impact of illness meanings on illness experience, arguing that the adverse emotional impact of mental illness attributions was related to perceived stigma, diminished selfesteem, and greater depressive symptomatology (Mechanic, McAlpine, Rosenfield \& Davis, 1994).

Another model that has influenced considerable research in public health, the health belief model of Marshall Becker, also considers lay explanatory beliefs, but with a more applied focus on recommended illness 
behaviors and appropriate utilization of health services (Rosenstock, Strecher \& Becker, 1988). The model considers people's own judgments about their susceptibility and the severity of their illness with reference to benefits of and barriers to treatment as determinants of illness behavior and help seeking. Theories of reasoned action from research in social psychology as well as the health belief model provide a rationale for health education to promote medically appropriate help seeking (Ajzen \& Fishbein, 1980; Kelly, Mamon \& Scott, 1987). Even in settings where they are clearly related, the dynamics of these relationships, which are especially important for applications in public health, may be highly complex (Calnan \& Rutter, 1988).

Anthropologists have criticized the limitations of the health belief model for social research because it regards beliefs only as barriers with reference to true medical knowledge of health professionals (Good, 1994). Byron Good argues that the health belief model is based on inadequate assumptions of a culturally invariant, utilitarian explanation of illness behavior (Good, 1986). Although Quah (1985) introduced a cultural dimension to the theory by specifying the role of ethnicity, gender, and religious affiliation, her revision has had relatively little impact on the large body of research based on the health belief model.

Attribution theory, developed by social and cognitive psychologists, also relates ideas about illness and clinical outcomes. It is the theoretical foundation on which Beck's cognitive therapy (Beck 1979) and Seligman's theory of learned helplessness (Abramson, Seligman \& Teasdale, 1987) are based. The revised learned helplessness theory classifies experience according to specified categories, distinguishing internal/external, stable/variable, or global/specific evaluations for good and bad events to predict various outcomes, including illness course and health status over long periods (Peterson, Seligman \& Vaillant, 1988).

Another branch of attribution theory considers patients' and doctors' illness attributions, associating their congruence with better treatment compliance (Hunt, Jordan \& Irwin, 1989; Gillespie \& Bradley, 1988). Clinical experience and research, however, including findings from an EMIC study of leprosy and its impact on mental health and treatment adherence in Bombay, suggest that inferences drawn from analysis of illness meanings and analysis of the congruence of clinicians' and patients' explanations of 
illness may be contradictory. This study, for which the first of the EMIC explanatory model interviews was developed, showed that explanatory models based on humoral attributions of cause were associated with better clinic attendance, even though biomedical perceived causes were not. The meaning of illness with reference to its context was a better predictor of clinically significant illness behavior than whether or not patients' ideas are consistent with biomedical theory (Weiss, Doongaji, Siddhartha, Wypij, Pathare, Bhatawdekar, Bhave, Sheth \& Fernandes, 1992).

A large body of research associated with transcultural psychiatry has compared epidemiological findings across cultures. Studies like the International Pilot study of Schizophrenia and the Determinants of Outcome study that followed from it were facilitated by a seminal contribution of Robins \& Guze (1970), which had profound effects on psychiatric diagnosis and the current nosology. Identifying five operational components of validity gave rise to methods, research, and a new way of specifying psychiatric disorders according to criteria-based diagnostic systems. The WHO-supported multicenter studies of schizophrenia began with operational definitions of the disorder and inquired about its frequency of occurrence in different regions as a way to demonstrate the validity of the diagnostic categories (WHO, 1973; WHO, 1979; Kulhara, 1994; Gupta, 1992). The argument was based on the premise that if rates and expression of illness were similar in diverse settings, then the disorders were coherent entities.

Medical anthropologists, however, questioned the cultural validity of such assertions. They demonstrated that without efforts to validate the meaning and usefulness of these categories and criteria locally, the indiscriminate application of diagnostic criteria that made sense in one place could result in category fallacies in others, misleading epidemiological reports, and inappropriate strategies for planning and treatment (Guarnaccia, Good \& Kleinman, 1990; Bravo, Canino, Rubio-Stipec \& Woodbury-Farina, 1991; Jenkins \& Karno, 1992; Kleinman, 1988a) . Recognizing this problem, the DSM-IV task force convened an advisory committee on culture and diagnosis to enhance the cultural dimensions of psychiatric diagnosis. Although a limited mandate for that committee limited its impact on DSM-IV, which disappointed many of its members (Stein, 1993; Littlewood, 1992; Mezzich, Fabrega \& Kleinman, 1992; Fabrega, 
1992), the committee has heralded a message for mental health professionals that "beliefs about mental disorder have real consequences for the behavior of patients, physicians, and society" (Mezzich, Fabrega, Kleinman \& Parron, 1996, p.xiil).

Kleinman's concern for the cultural validity of clinical practice appears to have been an important influence on his initial formulation of the illness explanatory model (Kleinman, Eisenberg \& Good, 1978). Because it also provided a useful framework for characterizing and comparing illness experience, many researchers found it provided a needed and useful guide. But this concept of illness explanatory model lacked operational precision, and systematic comparisons were difficult to make for many of the same reasons that motivated development of semi-structured interviews and led to their current popularity in psychiatric epidemiology and clinical research. The EMIC aimed to provide a method to reliably assess and compare explanatory models within and across groups, and to provide the foundation for a cultural epidemiology capable of analyzing relationships between illness-related beliefs and practices, and outcomes of practical clinical significance. Though important for research, these objectives were a matter of less concern in clinical practice, which focused more on assessment, empathy, and treatment, and for which the existing explanatory model framework served well as a heuristic to inculcate an appreciation for the interconnectedness of culture, health, and illness. Even as research activities focusing on comparative analysis of explanatory models had begun to develop, Kleinman's original interest in illness explanatory models had begun to shift from clinical applications to broader contexts of suffering, both within and beyond clinical settings, and his work continues to emphasize these interests (Desjarlais, Eisenberg, Good \& Kleinman, 1995; Kleinman, 1986; Kleinman \& Kleinman 1991). This led naturally to a focus on analysis of illness narratives (Kleinman, 1988b), rather than further development of the explanatory model framework as an analytic tool for a cultural epidemiology.

The lack of operational specificity in the monograph most frequently cited as Kleinman's elaboration of the explanatory model framework, Patients and Healers in the Context of Culture (Kleinman, 1980), appears to have been intentional. Although questions about treatment preference, cause, and course were all integral features of an explanatory model, the 
concept remained somewhat undifferentiated, a construct intended to cultivate a kind of ethnographic consciousness in clinical care. The style of the presentation reflected concerns that over-specifying the operational aspect of eliciting explanatory models, perhaps with a checklist or screening instrument, would undermine the sensitivity to culture he was trying to cultivate. The eight questions, which have been widely quoted and used as guidelines for eliciting explanatory models, were presented almost apologetically in a footnote (p.106n.), rather than under a featured heading or set of guidelines. While this approach was highly suitable for training clinicians and for bridging disciplines of psychiatry and anthropology via exploration of the clinical significance of illness meanings, it required further specification to serve as a foundation that could support a cultural epidemiology compelling enough to influence mainstream psychiatry, medicine, or public health.

The need for an operational formulation of the explanatory model framework that could meet this need became apparent to me and my colleagues from experience developing a comparative study of tropical disease and mental health at four centers in India in 1983 (Weiss, Sharma, Gaur, Sharma, Desai \& Doongaji, 1986; Behere, 1993). Efforts to specify clinical ethnographic features of explanatory models that were reliable, valid, and suitable for comparing clinical groups proved to be far more difficult than expected. Guided by Kleinman's contributions, we analyzed idioms of distress, perceptions of cause, and prior help seeking with reference to the local options in medically pluralistic settings. But experience in the interviews and efforts to summarize, analyze, and compare our findings, even from one interview to the next in the same clinic, raised serious questions about how to classify responses and accurately account for interactions between interviewers and subjects.

For example, in response to a question about the cause of their problem, one person might say it was from mixing hot and cold foods. Another person with the same idea might not say so when asked about the cause of the problem, but relate a similar account only when we asked pointedly whether the problem results from foods. Since people may hold more than one idea about the cause of their problems simultaneously without experiencing any conflict, a single-valued response variable was clearly inadequate. Also, the nature of a narrative indicated considerable 
variation in the degree of emphasis patients might place on a particular perceived cause. The same category of response could be a tentative idea that only first seemed plausible when the interviewer posed the question; or even if the subject had considered it before, it may have been mentioned only in passing or as a peripheral consideration. Alternatively, the account may have identified a definite idea without equivocation, and one among multiple responses could usually be identified as the most significant among the various perceived causes. The variations in the style of reporting and their relationship to the particular queries that elicited these ideas sparked questions for us about the meaning and validity of our findings and how we should classify and code responses. We had hoped to be able to compare explanatory models--or more precisely, components of explanatory models--with epidemiological, clinical, and outcome data to determine the practical significance of illness meanings. Although many of our concerns would not have been a problem for ethnographic study aiming to characterize the range of illness experiences in a community, they were serious problems that could subvert our aims to advance a cultural epidemiology to clarify interrelationships among components of explanatory models and their relationships to various clinical outcomes.

We also recognized that the solution was not merely to refine methods to classify, code, and quantify; no matter how sophisticated, either extreme along the spectrum of quantitative and qualitative approaches had certain limits in its capacity to answer the questions we and others in the field of cross-cultural psychiatry were asking about illness experience and its impact. Careful attention to both ethnographic context and epidemiological principles are essential and complementary. From our experience developing an explanatory model interview, we formulated the structure for the EMIC to elicit information needed for coding and comparing responses from large numbers of respondents and to provide a framework for eliciting narratives required for interpreting the meaning of categories and dynamic relationships. We also developed computer software applications for managing, cross-referencing, and working effectively with a mix of quantitative and qualitative data. 


\section{Structure of the EMIC}

The operational formulation of the illness explanatory model that emerged as we developed the EMIC is outlined in figure 1 . Patterns of distress refer to the full range of problems associated with a respondent's condition, including symptoms, social and economic disruptions, and stigma. Because stigma is such an important element of the distress associated with mental illness and some chronic medical conditions, and because it is an important influence on illness behavior and help seeking, most adaptations of the EMIC have elaborated elements of stigma pertinent to the particular sociocultural and clinical context in some detail. The way that distress and disorder lead to an experience of "spoiled identity," by which Goffman (1963) defines stigma, is essentially a matter of illness meaning. Failing to appreciate that, research on mental illness-related stigma restricted to studies of a limited range of potential correlates, like demographics and social class, provide too little information about its clinical significance or how policy should deal with it (Kleinman, Wang, Li, Cheng, Dai, Li \& Kleinman, 1995; Nuehring, 1979).

Perceived causes refer to ideas of patients that answer the questions of why and how they have been so affected. Perceptions of cause may also influence what people do about their problems, the choices they make among the various options for help seeking available to them, their expectations from treatment, and perceived quality of care. The structure of queries recognizes that help seeking and treatment should be considered with respect to a wide range of options, including family and informal providers, generalists and specialists practicing mainstream Western medicine, various traditional healers, and so forth. The particular categories for coding should reflect locally available options, which may be aggregated for analysis according to emic categories of providers (e.g., faith healers, Ayurvedic doctors, homeopaths) or according to etic categories based on priorities of the researchers (e.g., government, private, and nongovernmental health care services).

To understand the broader context of ideas about illness in general, which may clarify the meaning and implications of ideas and behavior concerning the particular illness being studied, a section on general illness beliefs may inquire about a range of conditions other than the problem affecting the subject. This indicates whether previous responses 
are illness-specific and how pluralistic a subject's ideas about health and illness actually are. Whether or not subjects in India refer to a magicoreligious or humoral basis for their problem, and whether or not they have used or have considered Ayurvedic or homeopathic treatment for it, questions about other selected diseases will indicate whether these ideas are significant in the broader context of the person's illness experience. For example, since Indian culture associates smallpox with a deity, regards Ayurveda as particularly effective for joint pains, and considers homeopathy to be especially suitable for treating asthma and allergies, questions about these conditions are likely to indicate whether ideas about illness not reported for the current condition figure in this person's experience, and if so, how.

The style of queries in EMIC interviews follows a similar pattern for each section (figure 2). Recognizing the potential influence of the professional setting and differences in perceived status of interviewer and respondent as potential sources of bias, the interviewer begins with an introduction that emphasizes to the subject that these queries are not test questions, that their answers will not be judged right or wrong, and that the right answers are the ones that best reflect the subject's own ideas and experience. Interviewers must be trained, and they must understand the aims of the interview and that a sensitive, empathic style is essential if subjects are to feel sufficiently at ease to discuss disturbing aspects of their lives and problems. Although no script can substitute for a sensitive interviewer, we have found that short vignettes and metaphors may help subjects to understand the aims of the study and enable them to be more candid. Brief scripted introductions at the beginning of each section and instructions to the interviewer to reinforce key points as needed aim to enable the respondent to speak freely. The following is extracted from the introduction to the section on perceived causes used in several EMIC interviews in India:

Each of us may explain something that happens in various ways. For example, if a farmer has a poor crop, he might explain it as due to bad soil, bad seeds, poor quality fertilizer, not enough water, something he has done, angry spirits or just fate. Similarly, people have different ideas about what hurts them or makes them sick. We would like to understand your 
ideas about your problem...

After introducing the section with an effort to counteract the intimidating influence of the clinic setting on a respondent's willingness to speak freely, the first queries are open-ended, for example, in the section focusing on patterns of distress: "What is it that troubles you about this problem?" and in the section on perceived causes: "What do you think is the cause of this problem?" Because a researcher cannot assume that failure to mention a particular response means that it is irrelevant, these open-ended queries are followed by a series of questions focusing on categories of particular interest in this study. In their respective sections of the interview these questions may focus on specific symptoms, elements of stigma, perceived causes, and preferred sources of help.

Since multiple responses from a single subject frequently result from these open-ended and screening questions, each section of the interview concludes with queries requesting a summary judgment. For example, these include questions about the most troubling aspect of the condition, that aspect of his or her condition a respondent noticed first, the most important perceived cause, the cause that occurred to a respondent after first identifying the problem, the most important source of help, the first source of help consulted after first becoming aware of the problem, and so forth. Summary questions, especially those referring to perceived causes, may also include queries for a retrospective account of how this aspect of the explanatory model has changed over time.

The data set that emerges from this semi-structured, in-depth interview has both quantitative and qualitative components. Responses are coded for quantitative analysis, and details in prose are maintained in a qualitative database that preserves the narrative context. Many questions in a particular explanatory model interview based on this framework must be adapted for the particular cultural group and clinical problem the investigator is studying. Information about appropriate codes for symptoms, perceived causes, help-seeking sources, relevant topics for the section on general illness beliefs, and the wording and specific choice of questions within each section may come from focus group discussions, key informants, ethnographic literature, and experience in the clinic and the community. Pilot testing is necessary to test and validate the questions and coding options. 
Data set and Analysis

Many questions in EMIC interviews are informative on their own, and when analyzing interview data, it is useful to examine tables and summary statistics at the outset. Techniques of data reduction help to analyze these complex data sets, and to answer some questions they may be essential. As discussed, each section includes a combination of open-ended queries, focused queries that screen for specific categories, and summary judgments that rank multiple responses according to various criteria (e.g., most important or first). Analysis variables for specified categories take into consideration whether a response (e.g., food as a perceived cause of the problem) was discussed in response to an open-ended query, whether it was reported only after probing, and whether it or something else was considered most important and/or first perceived cause. The overall emphasis on the significance of a particular category of perceived cause, symptom, or help-seeking option is based on which queries elicited that response over the course of the EMIC interview, and if it was elicited by a focused query about a particular category, whether it was mentioned or emphasized.

This approach makes it possible to attach a numeric weight corresponding to the degree of emphasis a respondent places on that category of response for perceived causes, symptoms, and help-seeking options. The relative weight attached to a response from each section of the interview (i.e., open-ended query, focused screening, and summary queries) is based on experience with prior EMIC interviews and aims of the current study. These derived analysis variables make it easier to summarize and compare findings. They also make it possible to analyze relationships between explanatory model variables and demographics, ethnicity, subcultural differences, and clinical data, such as diagnoses, psychometric scale scores, course of illness, treatment adherence, and outcome.

To illustrate, consider how we derived perceived cause (PC) analysis variables in EMIC studies of leprosy and depression in India. The PC analysis variables indicate the emphasis a respondent gave to each category. For example, identifying a particular PC as most important contributed a weight of 5 to the analysis variable; identifying it without prompting in response to the initial open-ended query contributed 4; 
identifying it as a definite cause in response to a screening question that inquires specifically about this cause contributed 3 or 2 (depending on whether it was emphasized as definite or only mentioned as a possibility); and identifying it as the first cause the subject considered after first becoming aware of the problem, regardless of whether it was currently considered a cause, contributed 1. Contributions from each of these three considerations were added, that is, whether it was most important, whether it was reported (mentioned or emphasized) in response to an open-ended or screening query, and whether it was identified as the first cause. Numeric values for each PC analysis variable, based on this strategy, can range from 0 (if the cause was not mentioned at all) to 10 (if the cause was mentioned initially [adding 4], as most important [adding 5], and first [adding 1]).

In some EMIC studies the number of perceived causes may be large. Although this makes coding more sensitive to local nuances and the emic emphasis, it may also result in small cell sizes that make statistical analysis difficult. A second level of data reduction, collapsing categories with similar meanings into summary group categories, is often useful, because the number of categories researchers specify for coding may be more than optimal for a particular analysis. This capability of associating categories of response in specified summary groups recognizes the value of considering alternative configurations of categories; it also solves problems arising when a coding strategy, formulated for the convenience of coding data in the interview, fails to distinguish unrelated or distantly related categories from closely related categories of meaning, as may be required in the analysis.

Derivation of these group summary categories proceeds by considering the maximum contribution from component categories for responses to openended, focused, and summary queries individually, and then adding the maximum value from components of that summary group. For example, a collapsed summary variable for humoral perceived causes (PC-sum humoral) incorporates contributions from separately coded categories, including PCwind, PC-bile, PC-phlegm, and PC-heat/cold. If any of the variables included in that group is mentioned in response to the open-ended initial query, this contributes a value of 4 to the PC-sum humoral variable; if any is identified as most important, this contributes 5, and so forth, as for 
the derivation of each of the larger set of PC analysis variables. Since they are derived according to the same strategy, these collapsed summary variables also have a numeric range from 0 to 10.

A single category or group summary category may be compared with others. Maintaining more differentiated category codes at the stage of data collection enables a researcher to regroup these variables in different ways, according to the questions of particular interest. For example, it may be useful to include alcohol in a group with food and drink, referring to ingested substances. For some questions, however, considering a hypothesis about the role of abused substances or guiltevoking behaviors, it may be useful to group PC-alcohol with smoking, drugs, sexual promiscuity, punishment for a previous deed, and karma. Sorcery refers both to culture-specific ideas about supernatural power and an interpersonal process by which one person invokes these magico-religious forces to hurt someone else. For testing hypotheses about the role of magico-religious ideas, sorcery may be combined with perceived causes that refer to the influence of demons and deities, reflecting emic criteria. To examine the role of social and interpersonal attributions of cause, however, sorcery might alternatively be combined with marital discord, problems at work, and other interpersonal conflicts, reflecting the researcher's etic configuration for studying the role of social and interpersonal processes. A coding strategy for gathering data that is based on locally relevant categories offers options for reconfiguring data and testing competing hypotheses based on both emic and etic frameworks.

Internal relationships may clarify the meaning of some responses. For example, we expected that some subjects might not be able to specify any categories of response for some aspects of the illness explanatory model, and so "cannot say" was included among the coding options. In the study of depression in Bangalore, however, a finding that a response of "cannot say" to questions about the cause was associated with a desire not to disclose the existence of the problem, based on questions in the section concerned with stigma. This suggested that "unwillingness" rather than "inability" to specify the cause may be a reasonable interpretation of that category in that data set.

Sections of EMIC interviews focusing on stigma lend themselves to data reduction by scaling. After verifying their internal consistency by 
computing Cronbach's alpha (Cronbach, 1951), questions serving as

indicators of stigma have been combined additively in a stigma scale. This facilitates an analysis of stigma with reference to other explanatory model variables (including derived group summary variables), clinical data, or other variables with a hypothesized relationship to stigma. Examples of these techniques for data reduction in the analysis of EMIC data have been discussed in more detail for studies of depression (Raguram, Weiss, Channabasavanna \& Devins, 1996) and onchocercal skin diseases (WHO/TDR, 1995).

Because these data sets may be complex, computer programs are necessary for managing coded variables and text. Such programs were first prepared for the initial EMIC research studies and adapted for others that followed. DataEase, Epi Info, and other database programs have been used for managing quantitative data. The entry programs include appropriate range and logic checks, and some also include a menu-driven facility for verification by double entry. Text-oriented database managers have been used for managing and analyzing qualitative data.

\section{Qualitative Analysis of Textual Data}

Practical applications of cultural research require not just recognition of significant associations but a clear understanding of the processes that are responsible for these associations. The development of effective, culturally relevant psychotherapies that acknowledge and work with patients' explanatory models also requires a qualitative understanding of illness experience (Kareem \& Littlewood, 1992). The extensive textual component of many EMIC datasets offers valuable opportunities for integrated and interactive analysis of quantitative relationships among explanatory model variables and qualitative textual data, which may be organized according to thematically coded text segments. The finding of an association among variables in the data set may raise questions as well as answer them; examining thematic content and its narrative context may explain the meaning and the nature of the dynamic processes that drive quantitative relationships.

Qualitative textual data from EMIC interviews may be associated with text codes specified by the question that elicits the response, or by making free-form coding judgments as the investigator reviews prose data, 
irrespective of the particular queries that elicited these data. We have used Textbase Alpha to manage and analyze qualitative data, but a variety of programs for this purpose are now available. Many of these, such as Atlas and Ethnograph, have been discussed in the growing body of literature on qualitative analysis (Weitzman \& Miles, 1995; Fischer, 1994; Tesch, 1990). In addition, a World Wide Web site also provides information and demonstration copies for some of them (URL, http: //www.soc.surrey.ac.uk/caqdas).

The researcher can use these programs to identify typical, exceptional, and other responses of particular interest. Line counts of relevant codes--that is, the number of lines of text for selected coded segments in each respondent's data file--and code counts may help to identify quickly and easily which patients have the most or least to say about a topic. Summary statistics for line counts, code counts, and word counts for each subject are easily computed, and these numbers may be imported into popular statistics programs such as sPss for quantitative analysis with reference to any other variables in the data set. Our experience indicates, however, that this quantitative transformation of qualitative data is less useful than the power these programs afford to access prose from selected segments in selected records. Without such aids for managing data, much of the richness of the qualitative data set may be wasted as the number of records increases.

By retrieving selected coded segments from selected subjects, investigators can better understand the meaning of categories, relationships, and their implications. Some balance is required to decide what level of detail should be coded for quantitative analysis or maintained solely in prose for qualitative study. For example, although food has been coded as a category of perceived cause in many of the EMIC studies, different respondents may have different ideas in mind. In the leprosy study in Bombay we found some were concerned that mixing foods that should not go together was the cause, others with having eaten forbidden foods, and others said their problem resulted from eating contaminated foods. As the analysis of coded EMIC variables proceeds, the investigator may examine the data set to clarify the meaning of widely reported or infrequently reported responses, and retrieve prose segments from selected records in the qualitative data set. Consider some of the possibilities: 
Cross-tabulations of quantitative categorical data have enabled us to identify patients from cells with many responses, and for whom prose detail is likely to clarify the dynamic meaning of a strong association. On the other hand, examining the prose from a cell with a small number of responses may clarify the nature of exceptions to the typical pattern, from which there may also be much to learn. In the study of depression and stigma with the EMIC at the National Institute for Mental Health and Neuro Sciences (NIMHANS), Bangalore, we found a positive relationship between stigma scores and Hamilton Depression Scale scores (Raguram, Weiss, Channabasavanna \& Devins, 1996). By selecting patients in the upper quartile of stigma and depression scores and studying the narrative content of their interviews, we learned how stigma and depression are related for these subjects. By studying narrative content from records of selected patients with high depression but low stigma scores, we learned how some patients remain untroubled by self-perceived stigma despite their depression. Such insights may provide a useful guide for therapy with other patients who have been less successful on their own in coping with adverse effects of stigma associated with their depression.

\section{Research with the EMIC}

The idea of the EMIC emerged over the course of fieldwork in a study of tropical diseases and mental illness in 1983 at four sites in India (Banaras Hindu University, Varanasi; Central Institute of Psychiatry, Ranchi; KEM Hospital, Bombay; and NIMHANS, Bangalore). The operational formulation of the explanatory model and plans to use it as a framework for locally distinctive but comparable interviews arose from a series of discussions with Dr. Sushrut Jadhav, with whom I was working in the tropical disease and mental health study at NIMHANS. Since then a number of colleagues have taken part in the process of adapting existing interviews, and having done so, many of them have made their work available to contribute to the further development of the EMIC (table 1).

A cultural study of leprosy and mental health at KEM Hospital, Bombay, produced the first of the explanatory model interviews based on the EMIC framework, and this interview was refined in pilot testing and studied for interrater reliability (Weiss, Doongaji, Siddhartha, Wypij, Pathare, Bhatawdekar, Bhave, Sheth \& Fernandes, 1992). Development and use of this 
interview was completed during fieldwork in Bombay from July 1988 to January 1989 and from November 1989 to May 1990. This study documented the substantial emotional and social distress associated with leprosy, which typically remains undiagnosed, untreated, and ignored in the clinic. Analysis of illness explanatory models of patients with an early stage of the disease clarified details of the relationship between illness meanings, emotional distress, and social disruption resulting from a diagnosis of leprosy. Findings also clarified the nature of cultural idioms of guilt and punishment (e.g., karma) as perceived causes of leprosy, which were associated with depression.

Addressing the clinical and public health problems arising from prematurely discontinued treatment, our analysis of clinic attendance with respect to perceived causes of leprosy resulted in an unexpected finding. We had expected better compliance among patients who share health professionals' ideas of infection, sanitation, and hygiene as causes of leprosy. To the contrary, patients who attributed their disease to a humoral imbalance, described in terms rooted in the indigenous traditional medical system, Ayurveda, were more likely than others to have the best clinic attendance records for leprosy treatment. Identification of the problem as medical was apparently more important to patients than the distinction between traditional medical and Western biomedical models. What might seem for health professionals to be competing ideologies were compatible and mutually supportive ideas for these patients, and our analysis of the role of cultural context explained why. This finding would have been difficult to detect without the systematic coding methods embodied in the EMIC and difficult to understand without access to the narrative accounts of illness meaning that were an integral component of the data set.

In the next major EMIC study in India this version was adapted for research with psychiatric outpatients screened positive for depression at NIMHANS in collaboration with Drs. R. Raguram and S.M. Channabasavanna (Raguram, Weiss, Channabasavanna \& Devins, 1996; Weiss, Raguram \& Channabasavanna, 1995; Channabasavanna, Raguram, Weiss, Parvathavardhini \& Thriveni, 1993). Depression and the broader class of neurotic disorders proved to be an especially suitable focus for developing cultural research with the EMIC in this study because of cultural variations in 
symptomatology, a need for culturally valid criteria to distinguish distress and disorder for these conditions, and recurring clinical questions about the complexity of interrelationships among depression, somatoform, and anxiety disorders.

The Bangalore study also documented the interrater reliability of key variables of the EMIC, confirming findings from the previous study in Bombay. Computations of a summary weighted kappa ranged from .75 (Channabasavanna, Raguram, Weiss, Parvathavardhini \& Thriveni, 1993) to .79 (Weiss, Raguram \& Channabasavanna, 1995), depending on how many key variables were included in the summary statistic. This study also found comparable levels of agreement in an interrater comparison study with the SCID (summary weighted kappa $=.68)$, Hamilton Depression Scale $(r=.80$ for the 24-item version), and Hamilton Anxiety Scale $(r=.66)$.

Deriving a stigma scale from EMIC data with good internal consistency (Cronbach's alpha $=.71$ ), we found stigma was positively related to depression and inversely related to somatization by analyzing the relationships of stigma scores to symptom significance ratings for depression and somatization--i.e., explanatory model variables derived for analysis, as explained above for the perceived cause variables. Hamilton Depression scores and stigma scale values were also strongly related $(r=.47, p=.0001)$. Comparing analysis variables for perceived causes with indicators of clinical status, we found that attributing one's problem to poor health or prior illness was associated with higher Hamilton anxiety scores, but not depression scores. Hamilton depression scores were inversely related to somatoform perceived causes, such as weakness and nerves, and positively related to social causes, sexual causes, and karma (Raguram, Weiss, Channabasavanna \& Devins, 1996).

A more recent effort to adapt the EMIC for research in India has been undertaken in collaboration with Dr. S.R. Parkar at the KEM Hospital, Bombay, to study deliberate self-harm and predictors of suicide. This interview focuses on the meanings of both the parasuicidal event and the social and psychological problems associated with it. Although the work began there, use of the EMIC has by no means been restricted to India. As an early and continuing collaborator in the development of the EMIC (Jadhav, 1986), Dr. Sushrut Jadhav adapted interviews from the studies in India to prepare an explanatory model interview he is using to study white 
British patients with depression in London, England. Like the previous studies, his research uses the SCID to make DSM diagnoses and the Hamilton scales to specify levels of depression and anxiety.

Prominent among other research activities associated with the EMIC is a collaborative study of neurasthenia and chronic fatigue syndrome. Led by Dr. Keh-Ming Lin, Director of the Center for the study of the Psychobiology of Ethnicity at the Harbor-UCLA Medical Center in Los Angeles, this project adapted the EMIC in two workshops, the first at the Chinese University of Hong Kong in 1992, and the second at the University of Hawaii in 1993, attended by collaborators from three East Asian, one Indian, and three North American centers. In addition to adapting EMIC interviews for the multicenter study at these workshops, Dr. Lin's group has also developed modules for neurasthenia and chronic fatigue syndrome for the Scheduled Clinical Interview for DSM-IV (SCID), and a similarly enhanced version of the SCL-90. Although both of these disorders refer to a similar pattern of symptomatology focusing on fatigue and weakness, the interviews examine distinctive culture-specific interpretations emphasizing biomedical and traditional Chinese medical concepts.

Although the EMIC was initially conceived as a method for research, experience with it has also served the interests of program building and training. A seminar in the cultural psychiatry program of the Toronto Hospital adapted the EMIC framework to develop an explanatory model interview for maintaining a cultural database in the program, for research, and for clinical training in cultural case formulations. Other colleagues have also adapted this interview for research and record keeping in a cultural health information system in three cities in India.

Because sociocultural health research can provide policy-relevant information for various health programs, interest in the EMIC has not been restricted to psychiatry. The potential for acquiring useful data concerning determinants of illness behavior, perceived quality of health care, and expectations from clinical services has aroused the interest of health planners. A group led by Dr. Chanpen Choprapawon adapted the EMIC for use in a village-based study of childhood diarrhea in central Thailand, considering the differences in findings from interviews in a village survey and interviews of mothers of children then suffering from diarrhea. The research examined use of oral rehydration solutions and determinants of 
other help-seeking activities (Choprapawon, Chunsutiwat, Kachondham, \& Weiss, 1991). Dr. S.N. Morankar adapted the interview and methods used in that research for a comparable study of childhood diarrhea in two villages of Maharashtra, India (Morankar, 1994).

A focus on illness experience, particularly patterns of distress, has helped to clarify the nature of illness burden, providing a useful alternative to the traditional indicators used more commonly in public health, such as mortality, morbidity, economic impact, and disability, which may be inadequate for conveying the magnitude of suffering arising from some diseases (Chen, Kleinman \& Ware, 1994). In 1993 investigators participating in an eight-center collaboration, supported by the wHO Special Programme for Research and Training in Tropical Diseases, adapted the EMIC to characterize the local meanings, illness burden, and illness behavior associated with onchocercal skin diseases in Nigeria, Ghana, Cameroon, Tanzania, and Uganda (WHO/TDR Pan-African Study Group, 1995) . Although some regions of West Africa, where river blindness is the principal manifestation of the disease, are benefited by the Onchocerciasis Control Programme, as many as 15 million people living elsewhere in subSaharan Africa are affected by the disease in regions where skin disease is the most common form. This study showed that, rather than skin lesions and depigmentation, itching, which may seem too trivial a symptom to motivate a concerted response, is the main concern among most affected persons. Itching for many was so severe and unrelenting that it led to self-injury and secondary infections from scratching with sticks and rocks, as well as stigma resulting from uncontrollable and socially unacceptable scratching. Severe emotional distress and, for some respondents, suicidal ideation were reported. Findings documented the illness burden and helped to mobilize support for the recently established African Program for Onchocerciasis Control, funded by a consortium of donor agencies led by the World Bank, to reduce the burden of onchocerciasis in 16 African countries (World Bank, 1995) .

Conclusion

As the distinctive nature and variety of studies associated with the EMIC makes clear, these explanatory model interviews are not ready-to-use research instruments like the Hamilton scales for depression and anxiety, 
the SCID, the Composite International Diagnostic Interview, or other instruments commonly used in epidemiological research. Use of the EMIC involves a process of becoming familiar with an operational formulation of the explanatory model framework, discussed in this review, and existing explanatory model interviews. The process of adapting existing interviews for a particular study involves decisions about areas of emphasis, level of detail, structure of questions, appropriate categories for coding, and an optimal mix of quantitative and qualitative data.

Insofar as the initial effort to develop the EMIC identified it as an interview catalogue, the project remains a work in progress. Such a catalogue is still needed as a guide for developing comparable explanatory model interviews informed by a growing body of experience, which provides a source of illustrative questions, strategies for coding, details of data management, and techniques for analysis. Until such a comprehensive version of the EMIC is available, this review may serve in its place as an account of the development and uses of explanatory model interviews associated with the EMIC.

\section{Acknowledgements}

Development of the EMIC has benefited greatly from the input and support of many colleagues, including those mentioned in table 1 . I am especially grateful to Dr. D.R. Doongaji, who was Honorary Professor and Head of the Department of Psychiatry at KEM Hospital, Bombay, and Dr. S.M. Channabasavanna, Director of NIMHANS, Bangalore, who enabled the first two major studies with the EMIC in India to proceed. The diligence of Dr. Sanjay Siddhartha made it possible to complete the study of leprosy in Bombay, and the clinical wisdom of Dr. R. Raguram enhanced every aspect of the study in Bangalore. Dr. Sushrut Jadhav played a major role in formulating ideas for developing the EMIC and several interviews. I also appreciate the guidance and support of Dr. Arthur Kleinman during my years at Harvard when this work began.

Research and fieldwork were funded by the American Institute of Indian Studies; the Fulbright Program of the U.S. Educational Foundation in India; the International Programs Office of the Alcohol, Drug Abuse, and Mental Health Administration; an NIMH Research Scientist Development Award (RSDA) MH00616; and an NIMH Small Grants Program Award MH47104. The 
Pacific Rim Foundation supported two international conferences to adapt the EMIC for comparative studies of neurasthenia and chronic fatigue syndrome in Asia and North America, and the Center for the study of the Psychobiology of Ethnicity at UCLA provided additional support for these studies. 
Bibliography

Abramson, L.Y., Seligman, M.E. \& Teasdale, J.D. (1987) . Learned helplessness in humans: Critique and reformulation. Journal of Abnormal Psychology, 87, 49-74.

Ajzen, I., and Fishbein, M. (1980). Understanding Attitudes and Predicting Social Behavior. Englewood Cliffs, NJ: Prentice Hall.

Beck, A. (1979). Cognitive Therapy of Depression. New York: Guilford Press.

Behere, P.B. (1993). Explanatory model of mental disorders in Indian psychiatric patients. Indian Journal of Behavioural Science, 1, 1114 .

Bravo, M., Canino, G.J., Rubio-Stipec, M. \& Woodbury-Farina, M. (1991). A cross-cultural adaptation of a psychiatric epidemiologic instrument: the diagnostic interview schedule's adaptation in Puerto Rico. Culture, Medicine and Psychiatry, 15, 1-18.

Calnan, M. \& Rutter, D.R. (1988). Do health beliefs predict health behaviour? A follow-up analysis of breast self-examination. Social Science and Medicine, 26, 463-465.

Channabasavanna, S.M., Raguram, R., Weiss, M.G., Parvathavardhini, R. \& Thriveni, M. (1993). Ethnography of psychiatric illness: a pilot study. NIMHANS Journal, 11, 1-10.

Chen, L.C., Kleinman, A. \& Ware, N.C. (1994). Health and Social Change in International Perspective. Harvard Series on Population and International Health. Boston: Department of Population and International Health, Harvard School of Public Health (distributed by Harvard University Press).

Choprapawon, C., Chunsutiwat, S., Kachondham, Y. \& Weiss, M.G. (1991). Cultural study of diarrheal illness in two villages of Central Thailand. Journal of Diarrhoeal Disease Research, 9, 204-212. Cronbach, L.J. (1951). Coefficient alpha and the internal structure of tests. Psychometrica, 16, 297-334.

Desjarlais, R., Eisenberg, L., Good, B.J. \& Kleinman, A. (Eds.) (1995). World Mental Health: Priorities, Problems, and Responses in LowIncome Countries. New York: Oxford University Press.

Dohrenwend, B.P. \& Dohrenwend, B.S. (1974). Social and cultural influences on psychopathology. Annual Review of Psychology, 25, 417-452 . 
Eisenberg, L. (1977). Disease and illness: distinctions between professional and popular ideas of sickness. Culture, Medicine and Psychiatry, 1, 9-23.

Eisenbruch, M. (1990). Classification of natural and supernatural causes of mental distress: development of a mental distress explanatory model questionnaire. Journal of Nervous and Mental Disease, 178, $712-719$.

Fabrega, H., Jr. (1992). Diagnosis interminable: toward a culturally sensitive DSM-IV (Commentary). Journal of Nervous and Mental Disease, $180,5-7$.

Fischer, M.D. (1994). Applications in Computing for Social Anthropologists. London: Routledge.

Gillespie, C.R. \& Bradley, C. (1988). Causal attributions of doctor and patients in a diabetes clinic. British Journal of Clinical Psychology, 27, 67-76.

Goffman, E. (1963). Notes on the Management of spoiled Identity. Englewood Cliffs, NJ: Prentice Hall.

Good, B.J. (1986). Explanatory models and care seeking: a critical account. In S. McHugh \& M. Vallis (Eds.), Illness Behavior: A Multidisciplinary Model (pp.161-172). New York: Plenum Press.

Good, B.J. (1994). Medicine, Rationality, and Experience: An Anthropological Perspective. New York: Cambridge University Press. Guarnaccia, P.J., Good, B.J. \& Kleinman, A. (1990). A critical review of epidemiological studies of Puerto Rican mental health. American Journal of Psychiatry, 147, 1449-1456.

Gupta, S. (1992). Cross-national differences in the frequency and outcome of schizophrenia: a comparison of five hypotheses. Social Psychiatry and Psychiatric Epidemiology, 27, 249-252.

Hahn, Robert (1984). Rethinking "Illness" and "Disease." Contributions to Asian Studies, Vol. 18: South Asian Systems of Healing, Edited by E.V. Daniel and J.F. Pugh. Leiden: Brill. Pages 1-23. Headland, T.N., Pike, K.L. \& Harris, M. (Eds.) (1990). Emics and Etics: The Insider/Outsider Debate. Frontiers of Anthropology Series, No. 7. Newbury Park: Sage Publications.

Hunt, L., Jordan, B. \& Irwin, S. (1989). Views of what's wrong: diagnosis and patients' concepts of illness. Social Science and Medicine, 28, 
945-956.

Jadhav, S. (1995). The ghostbusters of psychiatry. Lancet, 345, 808-810.

Jadhav, S. (1986). Help-seeking Behavior, Choice of Healers and

Explanatory Models. Thesis submitted to the University of Bangalore in partial fulfillment of the MD degree in Psychological Medicine.

Jenkins, J.H. \& Karno, M. (1992). The meaning of expressed emotion:

theoretical issues raised by cross-cultural research. American Journal of Psychiatry, 149, 9-21.

Kareem, J. \& Littlewood, R (Eds.) (1992). Intercultural Therapy. Oxford: Blackwell.

Kelly, G.R., Mamon, J.A. \& Scott, J.E. (1987). Utility of the Health Belief Model ni examining medication compliance among psychiatric outpatients. Social Science and Medicine, 25, 1205-1211.

Kleinman, A. (1980). Patients and Healers in the Context of Culture. Berkeley: University of California Press.

Kleinman, A. (1977). Depression, somatization and the "new cross-cultural psychiatry". Social Science and Medicine, 11, 3-10.

Kleinman, A. (1986). Social Origins of Distress and Disease: Depression, Neurasthenia, and Pain in Modern China. New Haven: Yale University Press.

Kleinman, A. (1988a). Rethinking Psychiatry: From Cultural Category to Personal Experience. New York: The Free Press.

Kleinman, A. (1988b). The Illness Narratives: Suffering, Healing and the Human Condition. New York: Basic Books.

Kleinman, A., Eisenberg, L. \& Good, B. (1978). Culture, illness and care. Annals of Internal Medicine, 12, 83-93.

Kleinman, A. \& Kleinman, J. (1991). Suffering and its professional transformation: toward an ethnography of interpersonal experience. Culture, Medicine, and Psychiatry, 15, 275-301.

Kleinman, A., Wang, W.Z., Li, S.C., Cheng, X.M., Dai, X.Y., Li, K.T. \& Kleinman, J. (1995). The social course of epilepsy: chronic illness as social experience in interior China. Social Science and Medicine, 40, 1319-1330.

Koegel, P. (1992). Through a different lens: an anthropological perspective on the homeless mentally ill. Culture, Medicine, and Psychiatry, 16, 1-22. 
Kraemer, H.C., Pruyn, J.P., Gibbons, R.D., Greenhouse, J.G.B., Grochocinski, V.J., Waternaux, C. \& Kupfer, D.J. (1987). Methodology in psychiatric research. Archives of General Psychiatry, 44, 1100-1106.

Kulhara, P. (1994). Outcome of schizophrenia: some transcultural observations with particular reference to developing countries. European Archives of Psychiatry and Clinical Neuroscience, 244, 227235.

Littlewood, R. (1991). Against pathology: the new psychiatry and its critics. British Journal of Psychiatry, 159, 696-702.

Littlewood, R. (1992). DSM-IV and culture: is the classification internationally valid? (Current Themes) Psychiatric Bulletin, 16, $257-261$.

Littlewood, R. \& Lipsedge, M. (1989). Aliens and Alienists, 2nd Edition. London: Unwin Hyman.

Mathieu, A. (1993). Medicalization of homelessness and the theatre of repression. Medical Anthropology Quarterly, 7, 170-184.

Mechanic, D. (1995). Health and illness behavior and patient-pracitioner relationships. Social Science and Medicine, 34, 1345-1350.

Mechanic, D., McAlpine, D., Rosenfield, S. \& Davis, D. (1994). Effects of illness attribution and depression on the quality of life among persons with serious mental illness. Social Science and Medicine, $39,155-164$

Mezzich, J.E., Fabrega, H. Jr., Kleinman, A., Parron, D.L. (1996). Culture and Psychiatric Diagnosis: A DSM-IV Perspective. Washington: American Psychiatric Press.

Mezzich, J.E., Fabrega, H. Jr. \& Kleinman, A. (1992). Cultural validity and DSM-IV (editorial). Journal of Nervous and Mental Disease, 180, 4 .

Morankar, S.N. (1994). Cultural Determinants in Diarrhoeas: A Study of Village Community. Thesis submitted to Faculty of Mental, Moral, and Social Sciences, University of Poona for the degree Doctor of Philosophy in Anthropology. Pune, India: University of Poona. Murdock, G.P. (1980). Theories of Illness. Pittsburgh: University of Pittsburgh Press.

Nuehring, E.M. (1979). Stigma and state hospital patients. American 
Journal of Orthopsychiatry, 49, 626-633.

Peterson, C., Seligman, M.E.\& Vaillant, G.E. (1988). Pessimistic explanatory style is a risk factor for physical illness: a thirtyfive-year longitudinal study. Journal of Personality and Social Psychology, 55, 23-27.

Pike, K.L. (1967) Language in Relation to a Unified Theory of the Structure of Human Behavior, 2nd Edition. (First Edition, 1954). The Hague: Mouton.

Pinder, R. (1994). Turning points and adaptations: one man's journey into chronic homelessness. Ethos, 22, 209-239.

Quah, S.R. (1985). The health belief model and preventive health behavior in Singapore. Social Science and Medicine, 21, 351-363.

Raguram, R., Weiss, M.G., Channabasavanna, S.M. \& Devins, G.M. (1996) Stigma, depression, and somatization: A report from South India. American Journal of Psychiatry, 153, 1043-1049.

Riskind, J.H., Beck, A.T., Berchick, R.J., Brown, G. \& Steer, R.A. (1987). Reliability of DSM-III diagnoses for major depression and generalized anxiety disorder using the structured clinical interview for DSM-III. Archives of General Psychiatry, 44, 817-820.

Robins, L.N., Wing, J., Wittchen, H.U., Helzer, H.E., Babor, T.F., Burke, J., Farmer, A., Jablenski, A., Pickens, R., Regier, D.A., Sartorius, N. \& Towle, L.H. (1988). The composite international diagnostic interview. Archives of General Psychiatry, 45, 1069-1077.

Robins, E. \& Guze, S.B. (1970). Establishment of diagnostic validity in psychiatric illness. American Journal of Psychiatry, 126, 983-987.

Rogler, L.H. (1989). The meaning of culturally sensitive research in mental health. American Journal of Psychiatry, 146, 296-303.

Rogler, L.H. (1992). The role of culture in mental health diagnosis: the need for programmatic research (Editorial). Journal of Nervous and Mental Disease, $180,745-747$.

Rosenstock, I.M., Strecher, V.J. \& Becker, M.H. (1988). Social learning theory and the Health Belief Model. Health Education Quarlerly, 15, $175-183$.

Rosenthal, R. (1991). Straighter from the source: alternative methods of researching homelessness. Urban Anthropology, 20, 109-126.

Sartorius, N. (1973). Culture and the epidemiology of depression. 
Psychiatrica Neurologia Neurochirugia, 76, 479-487.

Singer, M. (1995). Beyond the ivory tower: critical praxis in medical anthropology. Medical Anthropology Quarterly, 9, 80-106.

Stein, D.J. (1993). Cross-cultural psychiatry and the DSM-IV. Comprehensive Psychiatry, 34, 322-329.

Tesch, R. (1990). Qualitative research: analysis types and software tools. Bristol, PA: Falmer Press.

Weiss, M.G., Sharma, S.D., Gaur, R.K., Sharma, J.S., Desai, A. \& Doongaji, D.R. (1986). Traditional concepts of mental disorder among Indian psychiatric patients. Social Science and Medicine, 23, 379-386.

Weiss, M.G. \& Kleinman, A.K. (1988). Depression in cross-cultural perspective: developing a culturally informed model. In P. Dasen, N. Sartorius \& J. Berry (Eds.), Psychology, Culture and Health: Towards Applications (pp. 179-206). Beverly Hills: Sage.

Weiss, M.G., Doongaji, D.R., Siddhartha, S., Wypij, D., Pathare, S., Bhatawdekar, M., Bhave, A., Sheth, A. \& Fernandes, R. (1992). The explanatory model interview catalogue (EMIC): contribution to crosscultural research methods from a study of leprosy and mental health. British Journal of Psychiatry, $160,819-830$.

Weiss, M.G., Raguram, R. \& Channabasavanna, S.M. (1995). Cultural dimensions of psychiatric diagnosis: comparing DSM-III-R and illness explanatory models in South India. British Journal of Psychiatry, $166,353-539$.

Weitzman, E.A. \& Miles, M.B. (1995). Computer Programs for Qualitative Data Analysis. Thousand Oaks: Sage.

Williams, B.F. (1995). Public I/eye: conducting fieldwork to do homework on homelessness and begging in two U.S. cities. Current Anthropology, 36, 25-52.

World Health Organization (WHO). (1973). The International Pilot Study of Schizophrenia. Geneva: WHO.

World Health Organization (WHO). (1979). Schizophrenia: An International Follow-up Study. Chichester: John Wiley \& Sons.

WHO/TDR Pan-African Study Group on Onchocercal Skin Disease. (1995). The Importance of Onchocercal Skin Disease: Report of a Multi-Country Study. Applied Field Research Reports, No. 1. Geneva: WHO. World Bank. (1995). African Program for Onchocerciasis: Draft Program 
Document. Washington: World Bank Committee of Sponsoring Agencies. 
Figures and Table

Figure 1. Operational Formulation of Illness Explanatory Model for EMIC Interviews

Figure 2. Structure of EMIC Interviews

Table 1. Research with EMIC Explanatory Model Interviews 
Figure 1.

Operational Formulation of Illness Explanatory Model for EMIC Interviews

\section{Patterns of distress}

- Illness-related problems and concerns

- Name of illness, symptoms, anticipated outcome

- Psychological, social, and economic impact

- Stigma, disclosure, and self-esteem

- Marriage prospects and marital relations

\section{Perceived causes}

- Foods

- Psychological factors, psychosocial stressors and victimization

- Sanitation, hygiene, contamination, and health habits

- Infection, prior illness, constitutional factors

- Humoral imbalance

- Magico-religious forces

- Heredity

- Sexual experience, retribution for previous deeds

Help seeking and treatment

- Family support and home remedies

- $\quad$ Private practitioners and public clinics

- Western-styled health professionals, paraprofessionals, and specialists

- Traditional healers of various types

- $\quad$ Past experience and current preferences

General illness beliefs

- Explanatory models of illnesses other than current problem

- Focus on illnesses with a range of cultural meanings

- Relationship between mind and body

Disease-specific queries

- Ideas about the illness affecting the subject, but distinct from personal experience of presenting problems 
Figure 2.

\section{Structure of EMIC Interviews}

\section{Empowering introduction}

Explain the nature of the queries that follow and assure the respondent that responses will not be a source of humiliation.

\section{Open-ended queries}

By imposing minimal structure, enable the respondent to refer to what is most prominent in personal experience.

\section{Screening queries focused on categories of interest}

A relatively more structured section of the inquiry minimizes errors of omission. Respondents may not mention important aspects of illness experience in response to open-ended queries for various reasons. Without inquiring about specific categories of interest, one cannot assume that failure to mention a particular aspect of distress, perceived cause, or help-seeking experience means that these play no role in this person's experience.

\section{Summary judgment comparing multiple responses}

Since multiple responses are common, it is useful to weigh their relative significance, asking the respondent to identify which among them is most important, first in time (i.e., first symptom, first perceived cause, first help seeking), and how aspects of the explanatory model have changed over time.

\section{Prose elaboration}

Most questions in EMIC interviews elicit responses in the context of a narrative account of illness experience. This prose elaboration of various aspects of illness experience may be the principal interest for qualitative analysis of thematic content, or it may be consulted to clarify the meaning of coded categories. In either case, qualitative data constitutes an integral component of the data set. 